\title{
Update on the management of Barrett's esophagus in Austria
}

\author{
M. Riegler · I. Kristo · M. Nikolic · E. Rieder · S. F. Schoppmann
}

Received: 20 November 2017 / Accepted: 20 November 2017 / Published online: 4 December 2017 (C) The Author(s) 2017. This article is an open access publication.

\begin{abstract}
Summary
Background Barrett's esophagus (BE) is the premalignant manifestation of gastroesophageal reflux disease (GERD). Radiofrequency ablation (RFA) with and without endoscopic resection (ER) is a novel treatment for BE.

Methods Here we present a single-center update of the recommendations of a recent (June 2015) interdisciplinary expert panel meeting on the management of $\mathrm{BE}$ with dysplasia as well as cancer-positive and cancer-negative BE. We conducted a PubMed search of studies published in 2016 and 2017 on the topic of BE and RFA.

Results Our update reconfirms that BE positive for Tla cancer as well as low- and high-grade dysplasia justifies the use of RFA $\pm E R$, offering an $80-100 \%$ rate of BE clearance. RFA \pm ER of dysplastic BE is tenfold more effective for cancer prevention when compared with surveillance. Risk factors for recurrence and followup treatments include baseline histopathology (dysplasia/T1a cancer), esophagitis, hiatal hernia $>3 \mathrm{~cm}$, smoking habits, BE segments $>3 \mathrm{~cm}$, and $>10$ years of GERD symptoms. A baseline diagnosis for dysplasia and Tla cancer should include a second expert pathologist opinion. Recent data justify the use of RFA for nondysplastic BE only in controlled clinical trials. Antireflux surgery can be offered to those with function-test-proven, GERD-symptom-positive BE before, during, or after RFA \pm ER. Additionally, there is grow-
\end{abstract}

\section{Riegler}

Reflux Medical Vienna, Vienna, Austria

I. Kristo $\cdot$ M. Nikolic $\cdot$ E. Rieder ·

Univ.-Prof. Dr. S. F. Schoppmann, FACS ( $\bowtie)$

Department of Surgery, Upper-GI-Service, Comprehensive

Cancer Center, GET-Unit, Vienna General Hospital, Medical

University of Vienna, Vienna, Austria

sebastian.schoppmann@meduniwien.ac.at ing evidence that the intake of a sugar-rich diet is positively correlated with the development of GERD, BE, and cancer.

Conclusion RFA \pm ER should be offered for dysplastic $\mathrm{BE}$ and Tla cancer after ER as well as for nondysplastic BE with additional risk factors in controlled trials. Antireflux surgery can be offered to patients with function-test-proven GERD-symptom-positive BE. Diet considerations should be included in the management of GERD and BE.

Keywords Barrett's esophagus - Esophageal adenocarcinoma - Antireflux surgery - Esophageal manometry . Squamo-oxyntic gap - Cardiac mucosa - Low-carbohydrate diet

\section{Introduction}

Barrett's esophagus (BE) represents the morphologic premalignant manifestation of gastroesophageal reflux disease (GERD), which develops as a consequence of the dysfunction and failure of the antireflux mechanism within the lower end of the esophagus ([1]; Fig. 1). Via low- (LGD) and high-grade dysplasia (HGD), nondysplastic BE may progress toward adenocarcinoma of the esophagus (risk approx. $0.5 \%$ per year; [2, 3]; Fig. 2).

Radiofrequency ablation (RFA; Fig. 3) and endoscopic mucosal and submucosal resection are treatment modalities for the durable eradication of $\mathrm{BE}$, dysplasia, and early cancer and have been demonstrated to foster cancer prevention $[2,3]$.

A recent Austrian multidisciplinary expert panel meeting held in Vienna in June 2015 summarized recommendations for the management of BE [3]. Here we aim to summarize and update the recommendation by inclusion of relevant studies published in 2016 
until December 2017 from the viewpoint of a highvolume center.

\section{Methods}

Based on the recommendations of the recent expert panel meeting we conducted a search of PubMed and Scopus including the following keywords: Barrett's esophagus, endoscopy, endoscopic mucosal and submucosal resection, anti-reflux surgery, fundoplication, gastroesophageal reflux disease, histopathology, LINX lower esophageal sphincter augmentation system, radiofrequency ablation, and surveillance. The analysis included meta-analyses, review articles, and retrospective follow-up studies. Statistical analyses were not applied.

\section{Results}

Our search aimed to update the current data on to the diagnosis, therapy, and follow-up of patients with GERD and BE.

\section{Diagnosis of $B E$}

The diagnosis of BE is established by histopathology of endoscopic biopsies obtained from the esophagus and esophagogastric junction (EGJ), using the novel Chandrasoma classification [1]: The normal lining of the esophagus and the proximal stomach are stratified squamous and oxyntic mucosa, respectively [1]. Owing to the failure of the antireflux mechanism within the lower end of the esophagus, reflux occurs and stimulates the metaplasia of the squamous lined mucosa $[1,2]$. Thereafter, the columnar lined esophagus (CLE) develops and is interposed between the squamous lining of the esophagus and the oxyntic mucosa of the proximal stomach, i.e., this condition is termed the squamo-oxyntic gap (SOG) and represents the morphologic proof of GERD [1].

According to the Chandrasoma classification, CLE includes cardiac mucosa (CM: mucus-cell-only epithelium), oxyntocardiac mucosa (OCM: mixture of mucus and parietal cells within the subfoveolar region of the glands), nondysplastic BE (cardiac mucosa with goblet cells; intestinal metaplasia: IM), LGD, HGD, and cancer ([1, 3]; Fig. 2). Over time, further dysfunction of the antireflux mechanism aggravates the reflux and increases the length of the CLE, i. e., the length of the SOG. BE affects $20-30 \%$ of individuals with symptoms of GERD [2]. The diagnosis of LGD, HGD, and early cancer should be confirmed by an expert pathologist (second opinion; $[4,5]$ ).

\section{Endoscopic BE treatment}

Endoscopic therapies for the elimination of BE include endoscopic mucosal and submucosal resection for the removal of nodules and tumors within the
CLE [3]. RFA represents a modern endoscopic therapy for the removal of endoscopically flat visible CLE containing BE, LGD, HGD, and early cancer $([2,3]$, Figs. 2 and 3). The radiofrequency energy is applied to the CLE under endoscopic vision from self-sized balloon catheter-mounted electrodes (RFA 360; Fig. 3), an endoscope tip (RFA 90, 60), or working channelmounted electrodes ("eagle" device; [2, 3]).

\section{Management of dysplastic BE}

The interdisciplinary expert panel meeting recommended RFA \pm ER for Tla cancer, HGD, and LGD [3]. An accurate baseline diagnosis is of profound importance for the disease management [3]. Recent studies have confirmed these recommendations.

Duits et al. [4] retrospectively examined the effect of RFA for elimination of BE with LGD in 255 patients after 42 months (range: $25-61 ; 3-5$ years of followup; SURF trial data). During the follow-up, $18 \%$ of the patients (45/255) progressed to esophageal cancer.

The study showed the vital importance of accurate baseline histopathology for the assessment of risk for disease progression after the RFA therapy. As such, the odds for progression increased 8-13-fold, and 22-38fold, when baseline LGD (prior to RFA) was assessed by each of the pathologists or reconfirmed by all of them, respectively [4].

Guthikonda et al. [6] conducted a retrospective analysis of 306 patients who underwent RFA for the elimination of dysplastic BE between March 2006 and June 2015. At the first follow-up endoscopy $(<1$ year) after RFA elimination, BE and dysplasia were assessed in 85 and $88.9 \%$ of cases, respectively. In all, 218 patients continued with follow-up to assess disease recurrence. During a mean time to recurrence of 1.88 years, $24 \%(n=52)$ of the patients developed recurrence of $\mathrm{IM}$, this translates to an incidence of $9.6 \%$ per year for IM after RFA. Recurrences were assessed in biopsy samples obtained from the esophagus, cardia, and both in $63 \%, 33 \%$, and $4 \%$ of cases, respectively. Following repeated RFA, 58\% $(n=30)$ of those with recurrence were free from IM. Those positive for IM were enrolled in additional followup RFAs. During the study, four patients developed cancer. Risk factors for progression and development of cancer included baseline HGD and longer CLE segments.

Cameron et al. [7] retrospectively examined the recurrence rate following one to six RFA \pm endoscopic mucosa resection (EMR) sessions in 137 individuals for the treatment of BE low- (25\%), high-grade (54\%), and intramucosal adenocarcinoma (21\%; AIM dysplasia trial). Elimination of dysplasia and intestinal metaplasia was achieved in $88 \%, 92 \%, 100 \%$, and in $69 \%$, $74 \%$, and $81 \%$ of patients after 1,2 , and 3 years, respectively. Thus, Kaplan-Meier estimates were 58\%, $88 \%$, and $95 \%$ and $41 \%, 72 \%$, and $82 \%$ for dysplastic and nondysplastic BE after 1, 2, and 3 years, re- 
Fig. 1 Antegrade endoscopic images (a-d) of columnar lined esophagus distal to the endoscopic squamocolumnar junction (SCJ; arrows). Note the presence of typical geometric pattern of the columnar lining distal to the SCJ in a, typical for cardiac-type mucosa. Biopsies obtained from the SCJ in cases a-d were positive for Barrett's esophagus without dysplasia. Images obtained using Storz technology

Fig. 2 Histopathology of nondysplastic Barrett's esophagus (a, NDBE), low-grade dysplasia (b, $L G D)$, high-grade dysplasia (c, HGD), and (d) cancer. Yellow arrow in a indicates goblet-cell-positive mucosa adjacent to the squamocolumnar junction. Goblet cells are the hallmark for the diagnosis of Barrett's esophagus without dysplasia. H\&E stain, $\times 50$ in NDBE, LGD, cancer; $\times 100$ in HGD. (Courtesy of Prof. Fritz Wrba, Vienna)
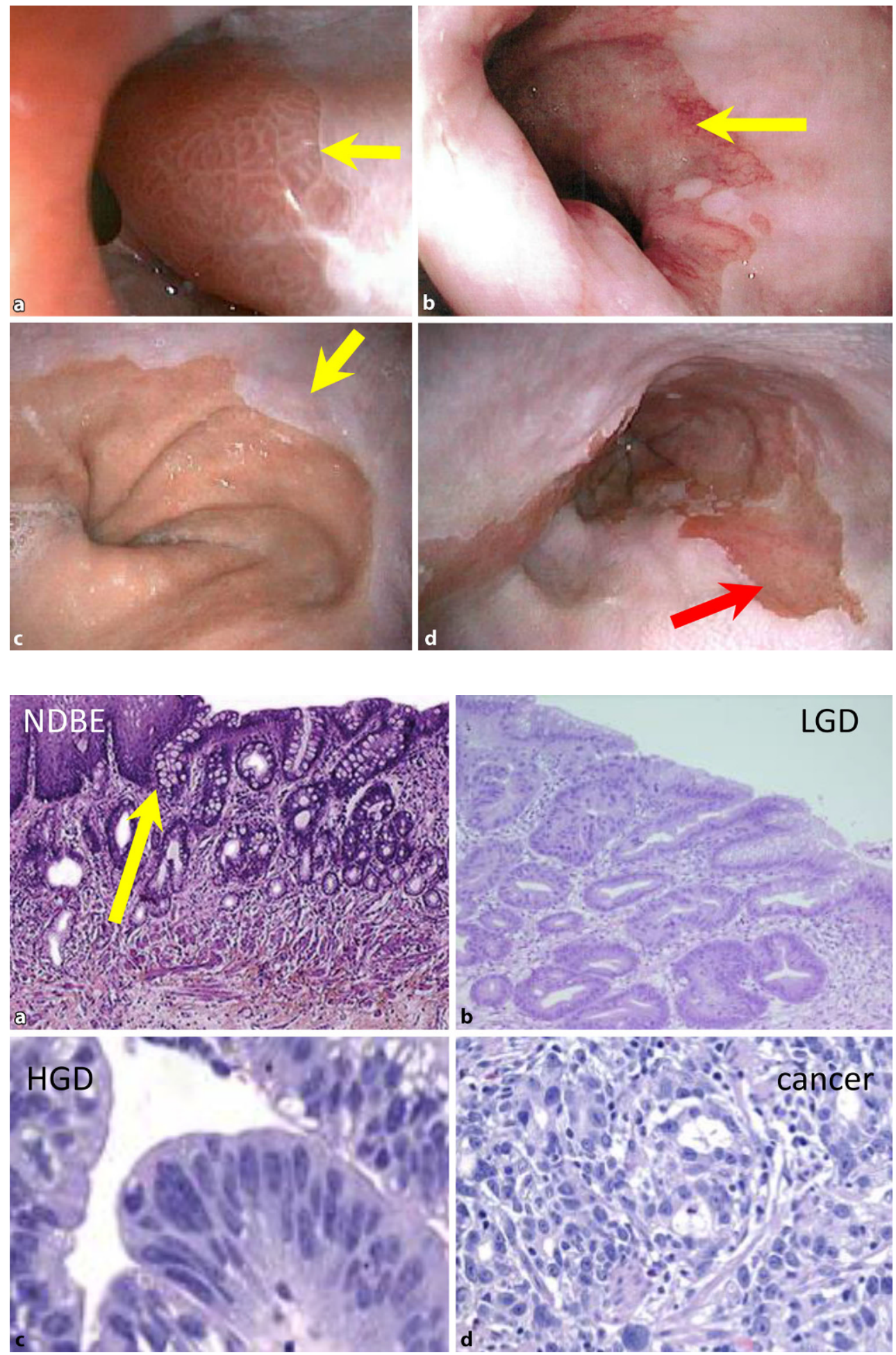

spectively. Dysplasia (HGD or LGD) was the most advanced stage of recurrence, and none of the patients progressed to or developed cancer. In all, $80 \%$ of the recurrences were assessed in biopsy samples obtained from the EGJ [7].

A recent meta-analysis examined the efficacy of RFA \pm EMR for the management of dysplastic and nondysplastic BE.
Luigiano et al. [8] confirmed that 1-3 years after RFA dysplastic and nondysplastic BE were eliminated in $60-100 \%$ and $60-90 \%$ of the cases, respectively. Furthermore, RFA was demonstrated to be superior to surveillance for the management of LGD. The cumulative 3-year risk of LGD to progress to HGD and cancer was $33 \%$ and $2.9 \%$ in the RFA \pm EMR treatment group vs. surveillance, respectively [8]. 

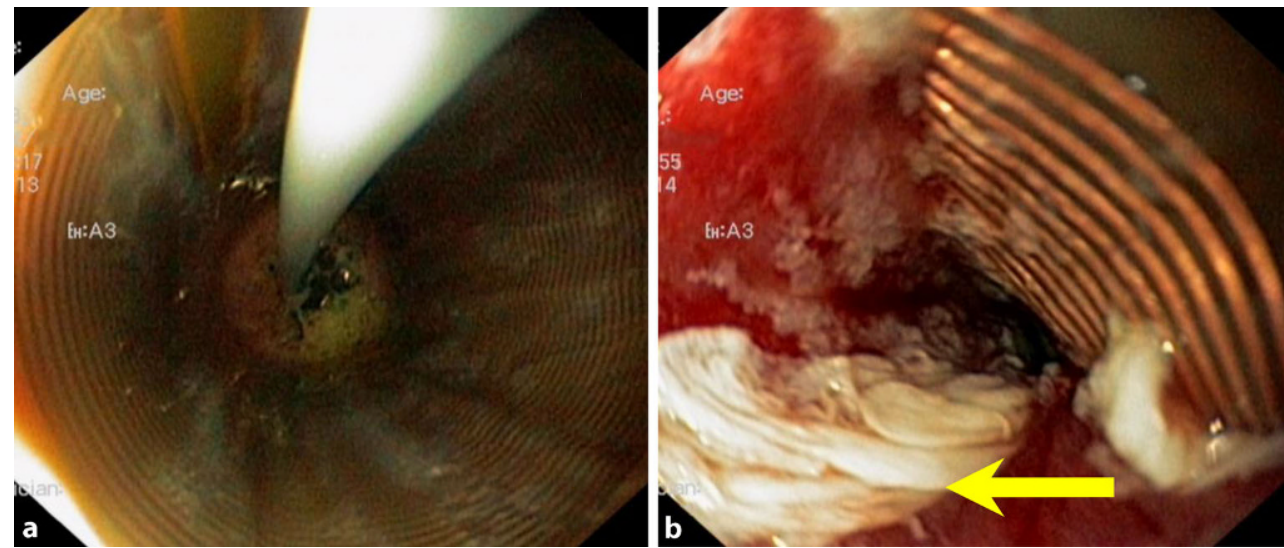

Fig. 3 Antegrade endoscopic view during RFA in the distal esophagus, using the catheter-mounted balloon (RFA 360). a View through the treatment balloon during the delivery of the radiofrequency energy to the tissue, as described in the text.

Following RFA $\pm \mathrm{EMR}$, the reported elimination rates for HGD and IM were $70-100 \%$ and $60-96 \%$, respectively [8].

In their meta-analysis, Fujii-Lau et al. [9] included 39 out of 3311 studies and examined the rate of recurrence of IM and dysplasia after RFA \pm EMR for the management of nondysplastic and dysplastic BE or early intramucosal cancer. The authors also compared the efficacy of RFA \pm EMR (RFA) vs. stepwise endoscopic treatment (SRER) for the management of dysplastic BE.

The pooled incidence for any recurrence, recurrence of IM, and dysplasia was 7.5/100 patient years (PY), 4.8/100 PY, and 2.0/100 PY, respectively. The recurrence rate differed between those who underwent SRER vs. RFA \pm EMR. The overall recurrence rate and the IM recurrence rate were increased after RFA \pm EMR vs. stepwise treatment: $8.6 / 100 \mathrm{PY}$ vs. $4.9 / 100 \mathrm{PY}$ and 5.8/100 PY vs. 3.3/100 PY for stepwise vs. RFA \pm EMR treatment, respectively [9].

The authors could not find a uniform typical risk profile for recurrent disease that was shared by all studies. However, factors fostering recurrence of $\mathrm{BE}$ after endoscopic treatment include esophagitis, presence of hiatal hernia and increased hernia size, CLE length, number of treatment sessions required to achieve IM-negative CLE, age, non-Caucasian background, smoking, decreased body mass index (25.3 vs. 29.8 for recurrence vs. no recurrence, respectively), and residual acidic reflux [9].

In their meta-analysis, Qumseya et al. [10] compared the efficacy of RFA vs. surveillance to prevent progression to HGD and cancer in patients with LGD BE. The final analysis included 19 out of 2029 cited studies. Calculations with fixed-effects models showed that RFA caused an $86 \%$ reduction in the risk of progression, when compared with surveillance. Including the data of 2746 patients, the random-effects models showed that the cumulative progression rate was $12.6 \%$ vs. $1.7 \%$ for surveillance vs. RFA, respec- b Image after RFA treatment with the balloon deflated. The yellow arrow indicates the ablated mucosal tissue. Note the red surface of the submucosa indicating adequate ablation

tively. Finally, the number of RFAs required to prevent one case of HGD or cancer was 9.2; thus, fewer than ten RFAs prevents one case of HGD or cancer in patients with LGD. Therefore, the data of the study by Qumseya et al. [10] reconfirm the recommendation of the recent expert panel meeting [3].

\section{Management of nondysplastic BE}

None of the studies explicitly examined the effect of RFA \pm ER on nondysplastic BE. However, the data on nondysplastic BE included in the aforementioned studies [4-10] support following the recent recommendation [3]. Thus, RFA should be offered to persons with $\mathrm{BE}$ and an increased cancer risk profile, which includes GERD for more than 10 years, hiatal hernia $>3.0 \mathrm{~cm}$, esophagitis, BE length, and history of dysplasia. However, at present, RFA for nondysplastic BE should be exclusively performed in controlled clinical trials.

\section{Antireflux surgery for BE}

The role of antireflux surgery and dietary aspects for the management of GERD and BE remains to be examined.

Conceptually, BE results from the impaired function or loss of function of the antireflux mechanism within the lower end of the esophagus. As a consequence, reflux occurs and fosters the development of GERD and BE [1]. Thus, it seems justified to consider the impact of treating the cause of the disease, e. g., repair of the antireflux mechanism (i.e., lower esophageal sphincter, diastasis of the musculature of the cura of the diaphragm, and formation of hiatal hernia). Medical therapy alters the acidity of the reflux, but does not alter the amount of reflux per se [11-13]. By contrast, Knight et al. [11] and Skrobic et al. [12] demonstrated that functional, effective antireflux surgery fosters the regression of BE and im- 
proves the efficacy of RFA in BE segments $>4.0 \mathrm{~cm}[11$, 12]. Thus, the recent data support antireflux surgery for symptomatic GERD and BE before, during, or after RFA [3, 13]. Esophageal manometry and reflux monitoring are recommended for an accurate diagnosis [2, $3,11,12]$.

Recent data showed that regular consumption of food and beverages rich in sugars and sweeteners positively correlates with obesity and GERD $[14,15]$. New data published in 2017 extended this notion to BE and cancer. Li et al. found a positive correlation between a sugar-rich diet and the development of BE [16] and cancer [17]. Sugar-rich nutrition increased the incidence of BE and cancer by $70-79 \%$ [16] and 51-58\% [17], respectively. Thus, nutrition seems to be of relevance in the development of GERD, BE, and cancer [14-17].

\section{Discussion}

Here, we provide an update of the recommendations of a recent interdisciplinary expert panel meeting on the management of BE held in 2015 [3].

Conceptually, endoscopic therapies aim to prevent the progression of $\mathrm{BE}$ to cancer. In line with this notion, the panel recommended RFA \pm EMR for BE with early cancer, HGD, and LGD [3]. This recommendation is supported by recent studies and meta-analyses published in 2016 and 2017 [4-10]. When compared with surveillance, RFA is significantly more effective in preventing the progression to cancer [10]. However, recurrence occurs in 10-25\% of the cases and warrants accurate surveillance [4, 6, 7]. Major risk factors for recurrence and progression to cancer after endoscopic therapy include markers of advanced stages of the disease including increased BE length $(>3 \mathrm{~cm})$, baseline diagnosis of dysplasia, esophagitis, and large hiatal hernia [4, 6-10]. In accordance with the recent recommendations [3], surveillance should be timed on the basis of the baseline histopathology and should be in 3- and 3-6-month intervals for HGD or early cancer and LGD, respectively. Follow-up RFA is recommended for the elimination of recurrent disease $[3,4,7,9,10]$.

There are discrepancies in the literature on how to manage nondysplastic BE [2]. Based on the recommendations of the expert panel, the published literature justifies RFA for nondysplastic BE within academic trials on patients with an increased cancer risk profile (GERD for more than 10 years, hiatal hernia $>3.0 \mathrm{~cm}$, esophagitis, BE length, and history of dysplasia; [3]). Recent studies focused on the treatment of dysplastic BE. However, these studies show that a higher stage of the disease (LGD, HGD) at baseline increases the probability for progression to cancer $[4,6,7]$. By contrast, RFA harbors a $90-100 \%$ chance for long-term elimination of nondysplastic $\mathrm{BE}$ [8]. Therefore, it seems reasonable to recommend RFA for nondysplastic $\mathrm{BE}$ in persons with an increased risk profile (as described earlier) in controlled academic trials [3]. After RFA, patients are kept on proton pump inhibitor (PPI) therapy [2].

Antireflux surgery targets the cause of the disease (impaired function of the antireflux mechanism, hiatal hernia) and stops increased exposure of the esophagus with the mediator of the disease (reflux), which in turn attacks the esophageal mucosa fostering the development of BE, dysplasia, and cancer [1, 11, 12]. In line with this view, a few new studies examined the effect of antireflux surgery on the treatment of BE. These studies showed that effective, functional antireflux surgery fosters regression of $\mathrm{BE}$ and support the efficacy of RFA [2, 11, 12]. These data are promising and warrant future studies to examine the efficacy and durability of endoscopic and surgical antireflux surgery for the management of BE and cancer prevention $[3,11]$. Currently, the literature supports offering antireflux surgery to patients with GERD-symptompositive BE before, during, or after RFA [2, 3, 11-13]. However, antireflux surgery should not be performed without an accurate diagnosis of esophageal function (manometry) and reflux monitoring [2, 3, 11-13].

Recent studies indicate the supportive role of diet and nutrition for the management of GERD [14, 16]. In 2017, this knowledge was extended to $\mathrm{BE}$ and esophageal cancer. In two studies, Li et al. [16, 17] demonstrated that the consumption of food and beverages containing sugars, sweeteners, and artificial sugars correlates with a 51-79\% increased risk for the development of $\mathrm{BE}$ and adenocarcinoma of the esophagus [16, 17]. Thus, it is justified to recommended the inclusion of a low-carbohydrate diet in the management of GERD and BE.

\section{Conclusion}

In summary, the endoscopic management (RFA \pm EMR) of dysplastic BE offers an accurate pretreatment diagnosis, is approximately tenfold superior to surveillance for cancer prevention [10], warrants accurate follow-up ( \pm RFA \pm endoscopic surgery; $[4,6]$ ), and should be conducted in expert specialized centers [3]. The efficacy of RFA for cancer prevention in nondysplastic BE should be tested in prospective academic studies. The outcome of studies investigating the impact of antireflux surgery before, during, and after RFA for cancer prevention is awaited $[2,3,11$, 12]. Finally, the recent literature supports the inclusion of a low-carbohydrate diet in the management of GERD and BE $[16,17]$. Therefore, the management of $\mathrm{BE}$ requires a well-orchestrated multidisciplinary approach for the benefit of our patients.

Acknowledgements The authors thank their patients, families, and friends for their positivity, passion, understanding, and support. Major gratitude is expressed to our friends and teachers Prof. Para Chandrasoma and Prof. Tom Demeester, who wisely opened our minds and motivated a thorough and 
responsible search for a deeper, holistic understanding of gastroesophageal reflux disease and Barrett's esophagus.

Funding Open access funding provided by Medical University of Vienna.

Conflict of interest M. Riegler, I. Kristo, M. Nikolic, E. Rieder, and S.F. Schoppmann declare that they have no competing interests.

Open Access This article is distributed under the terms of the Creative Commons Attribution 4.0 International License (http://creativecommons.org/licenses/by/4.0/), which permits unrestricted use, distribution, and reproduction in any medium, provided you give appropriate credit to the original author(s) and the source, provide a link to the Creative Commons license, and indicate if changes were made.

\section{References}

1. Chandrasoma PT. Metaplastic esophageal columnar epithelia in the squamooxyntic gap. In: GERD, a new understanding. New York, Boston, London, Oxford: Academic Press, Elsevier; 2017. pp. 82-6.

2. Kristo I, Schoppmann SF. Diagnosis and treatment of benign inflammatory esophageal diseases. Eur Surg. 2015;47(4):188.

3. Kristo I, Schoppmann SF, Riegler M, et al. Austrian panel recommendation for radiofrequency ablation of Barrett's esophagus. EurSurg. 2015;47(6):319-23.

4. Duits LC, van der Wel MJ, Cotton CC, et al. Patients with Barrett's esophagus and confirmed persistent low grade dysplasia are at increased risk for progression to neoplasia. Gastroenterology. 2017;152:993-1001.

5. Salomao MA, Lam-Himlin D, Pai RK. Substantial interobserver agreement in the diagnosis of dysplasia in Barrett esophagus upon review of a patient's entire set of biopsies. Am J Surg Pathol. 2017; https://doi.org/10.1097/PAS. 0000000000000988.

6. Guthikonda A, Cotton CC, Madanick RD, et al. Clinical outomces following recurrence of intestinal metaplasia after successful treatment of Barrett's esophagus with radiofrequency ablation. Am J Gastroenterol. 2017;112:87-94.

7. Cameron GR, Desmind PV, Jayasekera CS, et al. Recurrent intestinal metaplasia at the gastroesophageal junc- tion following endoscopic eradication of dysplastic Barrett's esophagus may not be benign. Endosc Int Open. 2016;04:E849-E58.

8. Luigiano C, Iabichino G, Eusebi LH, et al. Outcomes of radiofrequency ablation for dysplastic Barrett's esophagus: a comprehensive review. Gastroenterol Res Pract. 2016; https://doi.org/10.1155/2016/4249510.

9. Fujii-Lau LL, Cinnor B, Shaheen N, et al. Recurrence of intestinal metaplasia and early neoplasia after endoscopic eradication therapy for Barrett's esophagus: a systemic review and meta-analysis. Endosc Int Open. 2017;05:E430-49.

10. Qumseya BJ, Wani S, Gendy S, et al. Disease progression in Barrett's low grade dysplasia with radiofrequency ablation compared with surveillance: systematic review and meta-analysis. Am J Gastroenterol. 2017;112:849-65.

11. Knight BC, Devitt PG, Watson DI, et al. Long-term efficacy of laparoscopic antireflux surgery on regression of Barrett's esophagus using BRAVO wireless $\mathrm{pH}$ monitoring: a prospective clinical cohort study. Ann Surg. 2017;266(6):1000-5.

12. Skrobic O, Simic A, Radovanovic N, et al. Significance of Nissen fundoplication after endoscopic radiofrequency ablation of Barrett's esophagus. Surg Endosc. 2016;30(9):3802-7.

13. Subramanian CR, Triadafilopoulos G. Radiofrequencybased treatments for esophageal disease. Eur Surg. 2014;46(1):12-20.

14. Austin GL, Thiny MT, Westman EC, et al. A very lowcarbohydrate diet improves gastroesophageal reflux and its symptoms. Dig Dis Sci. 2006;51(8):1307-12.

15. Ma Der BXYR, et al. Body mass index is associated with Barrett's esophagus and cardiac mucosal metaplasia. Dig Dis Sci. 2006;51(9):1589-94.

16. Li N, Petrick JL, Steck SE, et al. Dietary sugar/starches intake and Barrett's esophagus: a pooled analysis. Eur J Epidemiol. 2017;32(11):1007-17. https://doi.org/10.1007/ s10654-017-0301-8.

17. Li N, Petrick LJ, Steck SE, et al. A pooled analysis of dietary sugar/carbohydrate intake and esophageal and gastric cardia adenocarcinoma incidence and survival in the USA. Int J. 2017; https://doi.org/10.1093/ije/dyx203. 